\title{
Rapid repetitive passive movement promotes knee proprioception in the elderly
}

\author{
Yan-Ying Ju • Jin-Kun Lin • Hsin-Yi Kathy Cheng • \\ Chih-Hsiu Cheng • Alice May-Kuen Wong
}

Received: 25 May 2012 / Accepted: 3 March 2013 / Published online: 3 April 2013

(C) European Group for Research into Elderly and Physical Activity (EGREPA) 2013

\begin{abstract}
Joint proprioception plays an important role in the generation of coordinated movements, maintenance of normal body posture, body conditioning, motor learning, and relearning. Previous studies have demonstrated that proprioceptive function of the elderly can be enhanced via
\end{abstract}

Prof. Yan-Ying Ju contributed to the study design, literature search, data collection and analysis, data interpretation and writing. Mr. JinKun Lin contributed to the literature search, data collection and analysis and figure preparation. Prof. Hsin-Yi Kathy Cheng contributed to the study design, literature search, data collection and analysis, data interpretation and writing. Prof. Chih-Hsiu Cheng contributed to the study design, data interpretation and writing. Dr. Alice May-Kuen Wong contributed to the study design, data collection and data interpretation.

\section{Y.-Y. Ju}

Department of Adapted Physical Education,

National Taiwan Sport University, 250 Wen-Hua 1st Rd,

Tao-Yuan, Taiwan

e-mail: yanju@mail.ntsu.edu.tw

\section{J.-K. Lin $\cdot$ C.-H. Cheng}

Department of Physical Therapy and Graduate Institute

of Rehabilitation Science, College of Medicine,

Chang Gung University,

259 Wen-Hua 1st Rd,

Tao-Yuan, Taiwan

J.-K. Lin

e-mail: clkhonk@hotmail.com

C.-H. Cheng

e-mail: chcheng@mail.cgu.edu.tw

\section{H.-Y. K. Cheng $(\square)$}

Graduate Institute of Early Intervention, College of Medicine, Chang Gung University, 259 Wen-Hua 1st Rd, Tao-Yuan, Taiwan e-mail: kcheng@mail.cgu.edu.tw

\section{A. M.-K. Wong}

Department of Physical Medicine and Rehabilitation, Chang Gung Memorial Hospital, Tao-Yuan, Taiwan

e-mail: walice@adm.cgmh.org.tw specific exercises; however, not all of the elderly can actively and safely participate in physical activities. The purpose of this study was to evaluate the effects of rapid repetitive passive movement in an average velocity of $90 \% \mathrm{~s}$ on knee proprioception in the elderly. A repeated-measure design was employed. Two age groups (12 young/12 elderly) were intervened with repetitive passive movements of 60 repetitions to the knee joint via two machines (isokinetic dynamometer/selfdesigned machine) in separate days. Changes in proprioception were evaluated via joint active repositioning and kinesthesia, both before and after the intervention. The results revealed that all subjects demonstrated good test-retest reliabilities on proprioceptive measurements. For the active repositioning and kinesthesia, significant statistical main effects were found for group and time, but not for machine. No statistical interactions were revealed. Despite of age-related proprioceptive declines, elderly subjects could benefit from rapid repetitive passive movements. This indicates that repetitive passive movement may be an alternative to physical activity for those who are home-bound, in a sedentary lifestyle, or with other health conditions that may limit their abilities to actively maintain or strengthen joint proprioception.

Keywords Older adults $\cdot$ Sense of position $\cdot$ Kinesthetic sense $\cdot$ Physical activity

\section{Introduction}

Proprioception is essential for people to accomplish daily tasks. It refers to the sense of knowing where one's body is in space and plays an important role in the generation of coordinated movements, maintenance of normal body posture, body conditioning, motor learning, and relearning [7]. Proprioception is primarily measured via joint position sense (static) and kinesthesia (motion) [9]. With the increase of age, knee proprioception is found to deteriorate [11, 32]. 
This deterioration can affect knee control and thus interfere with daily function such as balance [20, 21]. Therefore, proprioceptive training is needed in improving the quality of life in the elderly [10, 24, 37].

With global aging, issues of promoting health awareness and physical fitness of the elderly have become rather important. Although some exercises, such as tai-chi and wobble board exercise, were found to be beneficial to proprioceptive function among the elderly [33-36], not all of the elderly can actively and safely participate in these physical activities. In real-life situations, a lot of the elderly are homebound, bed-ridden temporarily because of recent surgery, or with other health conditions that might limit their abilities to resume physical activities. Alternative exercises which can improve their proprioceptive function need to be developed.

Repetitive passive joint movement (RPM), if provided to the subject's knee joint in a relatively high speed $(90 \%$ or $150 \%$ s) for about $30-60$ repetitions, was found to improve joint proprioception in healthy adults $[13,14]$. This improvement was observed in active repositioning and kinesthetic evaluation of the test limb. According to literature, the effects of rapid RPM on proprioceptive improvement suggested a possible promising treatment for joint proprioception $[8,26,28]$. In addition, research revealed that proprioception is the main predictor of balance in the elderly population [2, 15, 22, 31]. Whether the rapid RPM would improve proprioception in the elderly is not investigated.

To date, studies of rapid RPM were limited to healthy young adults, and thus similar studies should be conducted in the senior population. Comparing to the healthy young subjects, proprioceptive acuity of the elderly was found to deteriorate in both static $[1,11,16,34]$ and motion senses $[3,32]$. Because of the differences in proprioceptive sensitivity between the two age groups, it is unknown whether the elderly would demonstrate similar improvement as the healthy subjects did with rapid RPM intervention.

Therefore, the purpose of this study was to evaluate the effects of rapid RPM at a velocity of $90 \%$ s on knee proprioception in the elderly. Based on previous literature, passive mode provided by an isokinetic dynamometer (manual speed switch, $0-300 \%$ s; Cybex NORM Testing and Rehabilitation System, Computer Sports Medicine, Inc., Stoughton, MA, USA) was used to generate passive movement of the knee. However, the rather large and pricy isokinetic machine was not made for home use. A RPM machine which can move the knee joint in a peak angular velocity of $90 \% \mathrm{~s}$ was then designed and manufactured for subjects to use at home or at a group home. Therefore, another purpose of this study was to evaluate whether the young and old would respond differently to the two RPM machines - a commercialized isokinetic dynamometer and a self-designed RPM machine.

\section{Methods}

Experimental approach to the problem

This experimental study was part of a study series examining the effects of rapid RPM on knee proprioception [13, 14]. The main focus was to find out the difference between the young and the elderly in response to the rapid RPM. In addition, effects caused by different rapid RPM devices were investigated. A repeated-measure design was thus employed. The independent variable was the age group (young/elderly), RPM machine (isokinetic dynamometer/self-designed RPM), and time (pre-test/post-test). The influences of rapid RPM were evaluated via the dependent variables, including knee repositioning error for joint position sense and threshold to the detection of passive movement (TTDPM) for kinesthesia, measured both before and after movement interventions.

\section{Subjects}

Twelve healthy young volunteers and 12 healthy elderly participated in this study (Table 1). Subjects with a history that might interfere with the experiment, including (1) cognitive impairments with problem following commands, (2) knee injuries within the past 6 months, (3) severe osteoarthritis with marked swelling and range-of-motion limitation, (4) neuromuscular coordination impairments, (5) sensation deficits, and (6) currently taking pain killers or anesthetics, were excluded. All participants provided written informed consents. This study was approved by the Institutional Review Board of Chang Gung Memorial Hospital. All subjects were right leg dominant. The right leg was then designated as the testing leg.

All subjects demonstrated good test-retest reliabilities on proprioceptive measurements before movement intervention. For the young/elderly adults, same-day intraclass correlation coefficient (ICC) for active repositioning, TTDPM in flexion, and TTDPM in extension were $0.970 / 0.940$, $0.834 / 0.933$, and $0.799 / 0.828$, respectively. The over-day ICCs for the three measurement variables were $0.944 / 0.951$, $0.818 / 0.889$, and $0.879 / 0.832$, respectively.

\section{Equipment}

The equipments utilized in this study included an isokinetic dynamometer (Cybex NORM Testing and Rehabilitation System, Computer Sports Medicine, Inc., Stoughton, MA, USA) and a self-designed RPM device to provide rapid RPM to the knee joint at a peak angular velocity of $90 \%$, a high-speed camera (Basler A602f, Basler AG, Inc., Ahrensburg, Germany) along with an angle analysis software (MB-Ruler v.4.0, Markus Bader, Baden-Württemberg, 
Table 1 Subject demographics

\begin{tabular}{llllll}
\hline Group & $\begin{array}{l}\text { Number of subjects } \\
(\text { male/female) }\end{array}$ & $\begin{array}{l}\text { Mean age } \\
(\text { years })\end{array}$ & $\begin{array}{l}\text { Mean height } \\
(\mathrm{cm})\end{array}$ & $\begin{array}{l}\text { Mean weight } \\
(\mathrm{kg})\end{array}$ & $\begin{array}{l}\text { Average body } \\
\text { mass index }\left(\mathrm{kg} / \mathrm{m}^{2}\right)\end{array}$ \\
\hline Young & $8 / 4$ & $24.25(3.33)$ & $169.42(9.68)$ & $65.58(11.43)$ & $22.77(3.02)$ \\
Elderly & $3 / 9$ & $72.42(4.42)$ & $155.50(5.25)$ & $54.83(6.29)$ & $22.64(2.02)$ \\
\hline
\end{tabular}

${ }^{a}$ Statistically significant difference $(p<0.01)$

Germany) to capture knee joint range-of-motion via the positions of the reflective markers with a measurement accuracy of $99.17 \%$, and a device for the measurement of knee joint proprioception by moving the extremity to the desired angle (see Ju et al. [13] for details). The selfdesigned RPM device consisted of a rotary bar driven by a motor (5IK90RGU-AF, Honto Denki Gear Motor CO., LTD.; output $90 \mathrm{~W}$, range of speed setting 90-1,650 rounds per minute) with a speed-reducing gear (5GU50KB, Honto Denki Gear Motor CO., LTD., reduction ratio 50:1) mounted on a standing frame. One end of the rotary bar was attached to a rope, and the other end of it was attached to the leg plate mounted on a back-adjustable metal sitting frame. Pulleys were utilized to change the direction of pull via the rope. A control box was used to set the speed of the rotary bar at 30 rounds per minute. This whole rapid RPM unit moved the lower leg by pulling the leg plate with a peak angular deflection of $90 \% \mathrm{~s}$ (Fig. 1). The kinematic performance curves of the leg plate are shown in Fig. 2.

\section{Procedures}

The subjects were seated in a comfortable position, with their back fully supported by the backrest and legs hanging freely over the side of the metal frame. They were blindfolded and wore an earphone headset and an air splint on the lower leg to avoid feedbacks from sensory channels other than proprioception. Four reflective markers were attached to the greater trochanter, the iliotibial band level

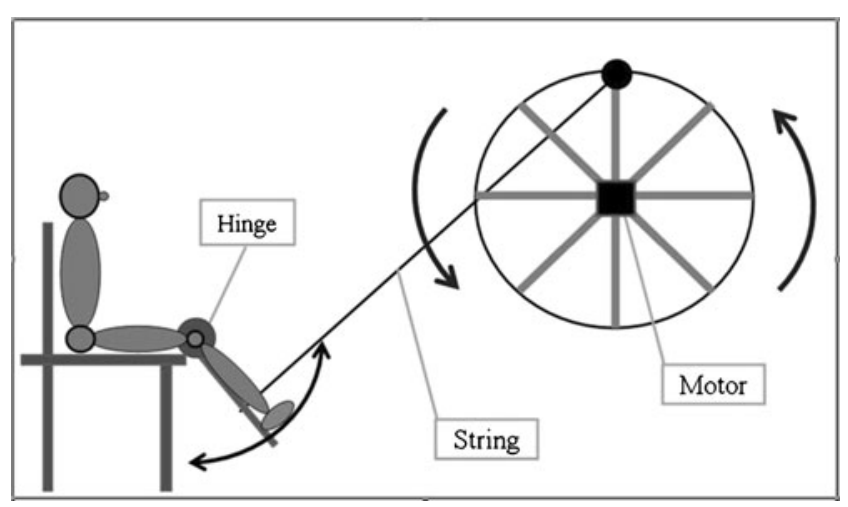

Fig. 1 Diagram of the self-designed RPM machine with the posterior crease of the knee when flexed to $80^{\circ}$, the head of the fibula, and the prominence of the lateral malleolus, respectively [30] (Fig. 3). High-speed camera was set at $1 \mathrm{~m}$ lateral to the side of the testing leg to capture the positions of the four markers in space for calculating angular changes of the knee. The frame rate of capture was set at $15 \mathrm{~Hz}$. The subject was given a hand-held switch and was instructed to press it down when he perceived movement of the extremity being tested.

Knee proprioception was measured via active repositioning error scores and TTDPM. Active repositioning was evaluated via the subject's ability to reproduce specific knee angles at $30^{\circ}, 50^{\circ}$, and $70^{\circ}$ flexion by random order. To start with, the subject's leg hung freely on the edge of the metal frame. From the starting position, the subject's leg was moved to a specific angle for $5 \mathrm{~s}$ and then returned to the starting position. After $5 \mathrm{~s}$, the subject was asked to reproduce that particular angle. The difference between the original starting angle and the reproduced angle was the error score. As for kinesthesia measurement, the starting angle of the knee was at $30^{\circ}, 50^{\circ}$, and $70^{\circ}$ flexion. The subject's leg was moved passively in an angular deflection of $0.5 \%$ to either flexion or extension. The subject was asked to press the on/off switch when he perceived the movement of the tested limb and to report the perceived movement direction. The difference between the angle while the subject pressed the switch and the starting angle was considered TTDPM. Two trials were conducted for each angle in both directions. A 20-s rest was provided between tests.

Following the measurement of proprioception, each subject received a RPM intervention of 60 repetitions. The subject's knee was set to move in the range of $10-110^{\circ}$. The peak velocity of the movement was $90 \%$. The subjects received RPM intervention from two different machinesan isokinetic dynamometer and the self-designed RPM machine - in two successive days. The order of the machine intervention was counterbalanced. In a previous study, stretch reflex responses observed via the electromyography signals of the subject's knee musculature were used for verification [14]. The same procedure was done and monitored in the elderly for the current study. After the movement intervention, the subject underwent knee active repositioning and kinesthesia testing again for two trials, the same as the pre-test protocol. 
Fig. 2 Kinematic performance curves of the leg plate during intervention. The $y$-axis represents knee extension and flexion angles

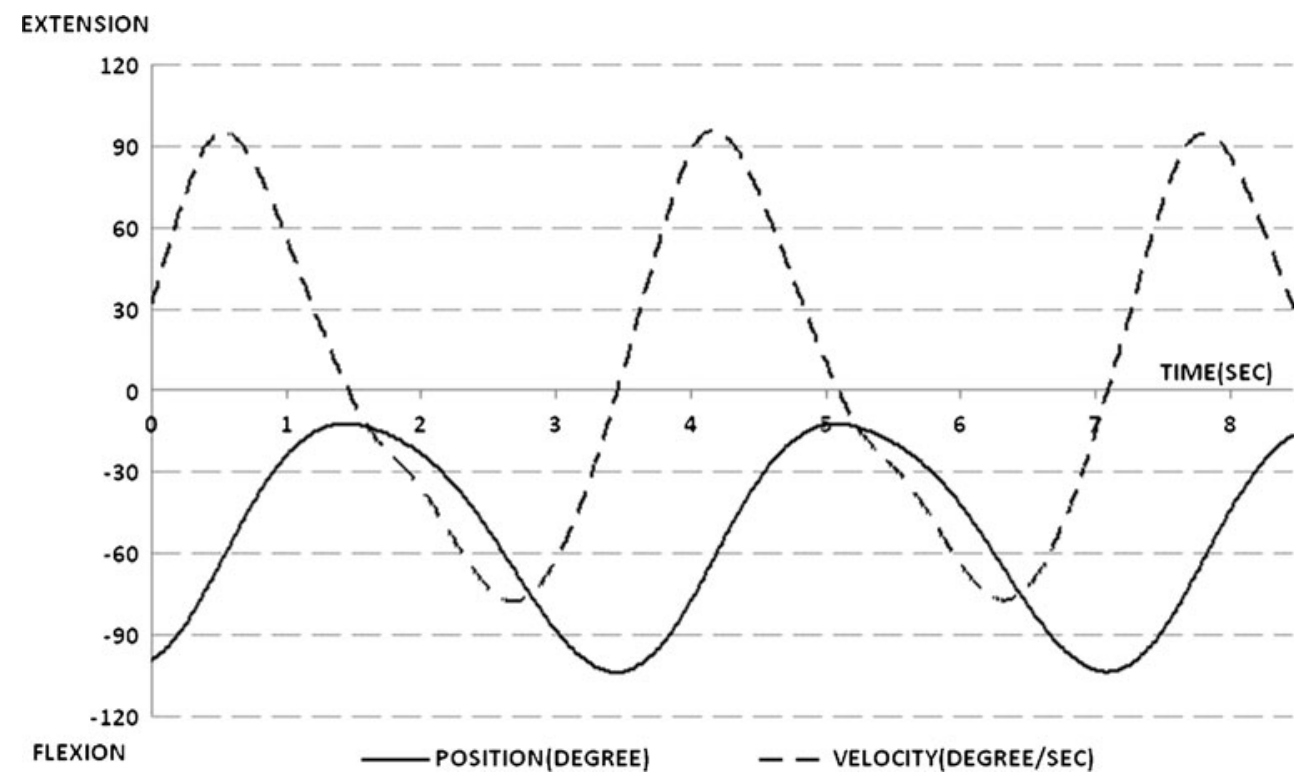

Results

Effects of rapid RPM on knee active repositioning

The absolute error scores for knee active repositioning before and after the intervention for two groups with two machines are shown in Fig. 4. Prior to the movement intervention, the repositioning errors for the young/elderly were $3.68^{\circ}(\mathrm{SD}=$ $\left.1.27^{\circ}\right) / 5.21^{\circ}\left(\mathrm{SD}=1.80^{\circ}\right)$ with the isokinetic dynamometer and were $3.63^{\circ}\left(\mathrm{SD}=0.95^{\circ}\right) / 5.15^{\circ}\left(\mathrm{SD}=1.35^{\circ}\right)$ with the selfdesigned machine. After the movement intervention, the repositioning errors for the young/the elderly were $2.81^{\circ}$ $\left(\mathrm{SD}=0.98^{\circ}\right) / 4.13^{\circ}\left(\mathrm{SD}=0.99^{\circ}\right)$ with the isokinetic dynamometer and were $3.06^{\circ}\left(\mathrm{SD}=0.99^{\circ}\right) / 4.11^{\circ}\left(\mathrm{SD}=0.85^{\circ}\right)$ with the self-designed machine. Significant statistical main effects were found for group and time $(p<0.001)$, but not for machine. No significant interactions were revealed. In general,

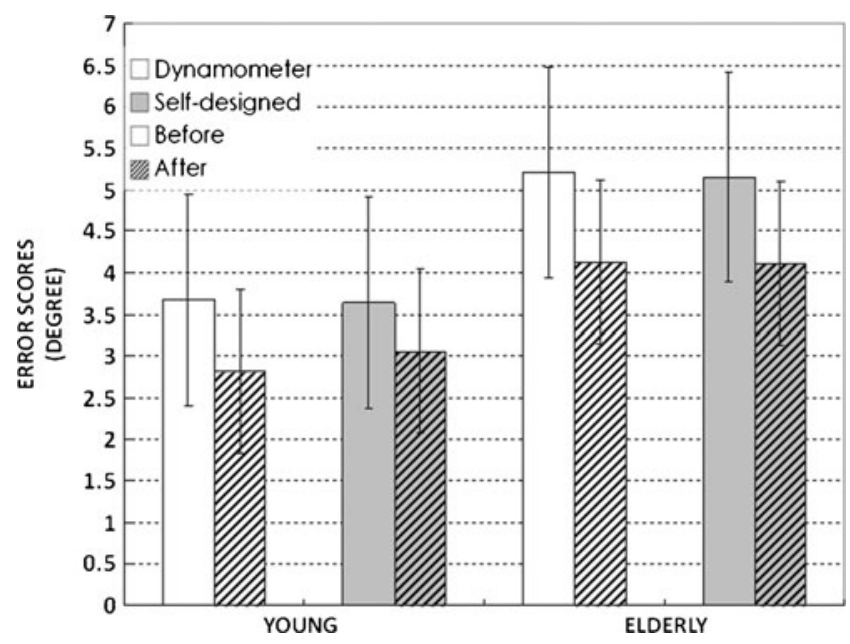

Fig. 4 Absolute error scores for knee active repositioning before and after the intervention 
the elderly demonstrated more errors in joint repositioning and relatively higher variability than the young.

Effects of rapid RPM on knee kinesthesia

The TTDPMs for knee kinesthesia in flexion and extension are shown in Fig. 5a, b. For measurements with knee flexion, prior to the rapid RPM intervention, the TTDPMs for the young and the elderly were $1.04^{\circ}\left(\mathrm{SD}=0.47^{\circ}\right) / 1.89^{\circ}(\mathrm{SD}$ $=0.81^{\circ}$ ) with the isokinetic dynamometer and were $1.05^{\circ}$ $\left(\mathrm{SD}=0.45^{\circ}\right) / 1.73^{\circ}\left(\mathrm{SD}=0.77^{\circ}\right)$ with the self-designed machine. After the movement intervention, the TTDPMs for the young and the elderly were $0.59^{\circ}\left(\mathrm{SD}=0.38^{\circ}\right) / 1.44^{\circ}(\mathrm{SD}$ $=0.76^{\circ}$ ) with the isokinetic dynamometer and were $0.52^{\circ}$ $\left(\mathrm{SD}=0.22^{\circ}\right) / 1.23^{\circ}\left(\mathrm{SD}=0.42^{\circ}\right)$ with the self-designed machine. Significant main effects were found for group and time $(p<0.001)$, but not for machine. No significant interactions were revealed.
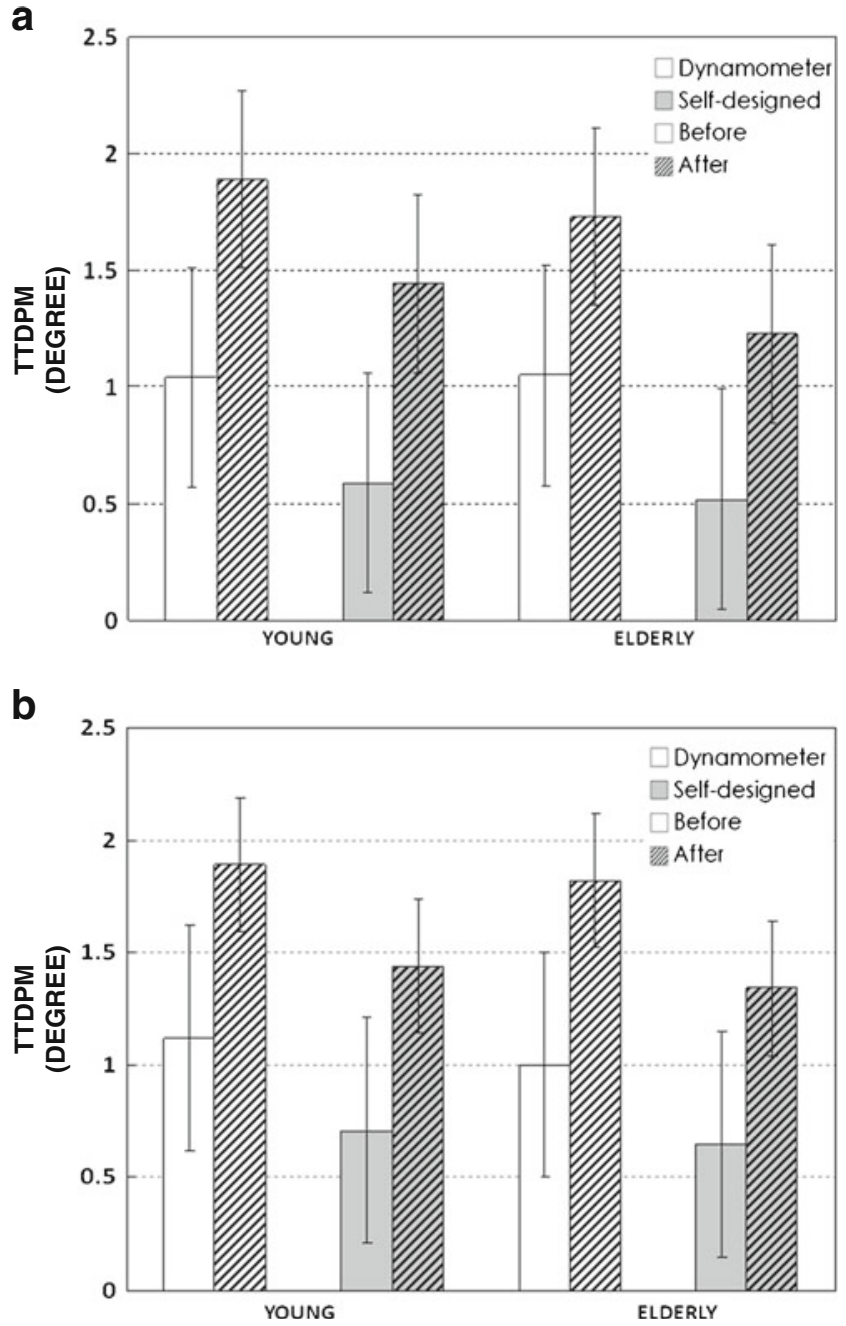

Fig. 5 TTDPMs for knee kinesthesia before and after the intervention $\mathbf{a}$ in flexion and $\mathbf{b}$ in extension
For measurements with knee extension, prior to the rapid RPM intervention, the TTDPMs for the young and the elderly were $1.12^{\circ}\left(\mathrm{SD}=0.50^{\circ}\right) / 1.89^{\circ}\left(\mathrm{SD}=0.55^{\circ}\right)$ with the isokinetic dynamometer and were $1.00^{\circ}\left(\mathrm{SD}=0.26^{\circ}\right) / 1.82^{\circ}$ $\left(\mathrm{SD}=0.65^{\circ}\right)$ with the self-designed machine. After the movement intervention, the TTDPMs for the young and the elderly were $0.71^{\circ}\left(\mathrm{SD}=0.30^{\circ}\right) / 1.44^{\circ}\left(\mathrm{SD}=0.63^{\circ}\right)$ with the isokinetic dynamometer and were $0.65^{\circ}\left(\mathrm{SD}=0.25^{\circ}\right) / 1.34^{\circ}$ $\left(\mathrm{SD}=0.47^{\circ}\right)$ with the self-designed machine. Significant main effects were found for group and time $(p<0.001)$, but not for machine. No significant interactions were revealed. For both flexion and extension, the elderly demonstrated higher thresholds to detect joint motion and more variability than the young.

The standard error of measurements (SEMs) for knee active repositioning, knee kinesthesia in flexion, and knee kinesthesia in extension were $0.155,0.074$, and 0.061 , respectively. The $95 \%$ confidence intervals of the reported SEMs for the three measurement variables were 3.659$4.284,1.037-1.335$, and $1.123-1.371$, respectively. The values of SEMs revealed that the differences of our proprioceptive measurement were detectable.

\section{Discussion}

Our results indicated that rapid RPM was able to improve a subject's proprioception based on the statistical significance revealed in time main effect. A large quantity of studies have disclosed the link between proprioception and balance $[11,21,23,25,33]$ or proprioception and daily motor tasks such as stair ascending and descending [11]. The elderly demonstrated deficits in sensorimotor performance. Poor proprioception deteriorates their control of movement execution and physical activity participation. The improvement in proprioception should be beneficial to the elderly in performing daily tasks. In addition, no group $\times$ time interaction was found; therefore, young subjects did not improve more than the elderly and vice versa.

This proprioceptive improvement after receiving rapid RPM might be due to a couple of mechanisms. First, since the rapid RPM improved both active repositioning and kinesthesia, the sensibility of the mechanoreceptors might have been enhanced. During the rapid RPM, muscles surrounding the knee were stretched, and these stretches would stimulate muscle receptors. Similar to the young, stretch reflex responses of the elderly were clearly shown at the end range of knee flexion and extension. These reflexes might activate the Ia afferents within the muscle spindle and further modulate the rubrospinal and rubro-bulbospinal pathways, leading to increased fusimotor activities via gamma system recruitment $[4,6]$.

Second, cyclic passive movement was found to decrease muscle stiffness, especially with increased movement 
velocity [26, 28]. According to Loram et al. [19], proprioception of movement is mainly sensed by muscle spindles registering changes in fascicle length. Short-range muscle stiffness reduces the transmission of joint rotations to the extrafusal fibers, leading to a proprioceptive dead zone for small movements. Based on this rationale, the transmission of joint rotation information would be less interfered with decreased muscle stiffness, consequently avoiding the proprioceptive dead zone.

Even though proprioception in the elderly was found to improve after rapid RPM, the underlying mechanisms that led to such improvement were still unrevealed. According to the literature, the neurophysiological changes of age-related proprioception declines include but are not limited to decreased spindle diameter [17], fewer intrafusal fibers [18], and decreased mean density of cutaneous receptors per unit of skin [12]. Results from this study only demonstrated that with these age-related proprioceptive declines, elderly subjects could still benefit from rapid RPM. Neurophysiological monitoring of the proprioceptors during clinical experimental studies would provide a more detailed explanation to the underlying mechanism.

Furthermore, no machine main effect was found. This indicated that the self-designed device could provide desired the RPM to the extremity and produce similar effects. Since rapid RPM leads to instant proprioceptive improvement and a dynamometer is not a cost-effective machine to use in household, our machine has the potential to become a therapeutic device, given further clinical and research supports. The results hopefully can facilitate the commercial design of such equipment. Future optimization of suchlike device, such as increasing the robustness of the motor performance, noise reduction, and outlook enhancement, should be accomplished.

To date, most evidences revealed that physical activities and trainings could improve proprioceptive acuity, whereas only very few investigated on passive movements. For example, Tsang and Hui-Chan [33, 34] studied the effects of tai-chi, a traditional Chinese martial art, and found that tai-chi practitioners performed better in passive knee joint repositioning. Waddington and Adams [35] found that a 5week wobble board training program was effective in improving ankle movement discrimination capability in 20 elderly subjects. Another study done by Westlake et al. [36] evaluated the effects of an 8-week balance exercise on ankle proprioception among the elderly and found that this exercise improved the subject's ability in velocity discrimination. Further studies on passive movement and its effect on proprioception in the elderly are needed.

With world aging and the increase in disability rate among aging population [27], finding alternatives for physical activities to promote healthy aging is of great importance. For elder adults with physical or cognitive conditions that might hinder their activity participation or jeopardize their safety during active participation, alternative exercises are needed in order to maintain their physical health. Based on the results of this study, rapid RPM could be a great alternative for these individuals. Daily use of rapid RPM at home, at the nursing home, or in the hospital might help in maintaining or improving proprioceptive functions in the elderly.

There are some limitations in this study. First, the post-test data of the current study were collected immediately following the intervention. No retention data were collected at this point. The long-term effects of RPM onto proprioception in the elderly need further investigation. Second, although the results of proprioceptive measurements were significant, the differences were small and it could be questioned if they had any functional relevance. Small-angle differences with statistical significance were also found in other studies. For example, Fischer-Rasmussen and Jensen [5] and Pap et al. [29] both investigated anterior cruciate ligament injured knee versus normal knee and found significant differences in joint angle of around $\pm 1^{\circ}$ between the injured and the normal knees. As for functional relevance, Tsang and Hui-Chan [33] detected a small difference of $1.9^{\circ}$ in knee joint repositioning between their tai-chi practitioners $(\mathrm{M}=69.4$ years, $\mathrm{SD}=5.5)$ and the control $(\mathrm{M}=72.3$ years, $\mathrm{SD}=6.1)$. Correlated with the limit of stability performance, the tai-chi practitioners indeed demonstrated significantly better reaction time in initiating voluntary weight shifting than the control group, which indicated that such a small change could be functionally relevant. Nonetheless, further investigations are needed to discover whether proprioceptive enhancement after RPM intervention could lead to any difference in functional activities.

\section{Practical applications}

Rapid RPM as used in this study was an intervention that led to instant proprioceptive improvement in the elderly, regardless of their age-related proprioceptive declines. It would be an alternative for physical activity to the elderly who are in a sedentary lifestyle, and it has the potential to become a therapeutic and conditioning device with further clinical and research evidence. Results in this study have enhanced our knowledge on knee injury prevention, treatment, and rehabilitation in the elderly. More research is needed to support the presumed mechanisms which explain such proprioceptive improvement.

\section{Disclosures}

Conflict of interest Yan-Ying Ju, Jin-Kun Lin, Hsin-Yi Kathy Cheng, Chih-Hsiu Cheng and Alice May-Kuen Wong declare that they have no conflict of interest.

Informed consent All procedures followed were in accordance with the ethical standards of the responsible committee on human experimentation (institutional and national) and with the Helsinki Declaration of 1975, as revised in 2000 (5). Informed consent was obtained from all patients for being included in the study. 
Human \& animal studies Not applicable.

Ethical statement This manuscript is original and not previously published, nor is it being considered elsewhere until a decision is made as to its acceptability by the EURAPA Editorial Review Board.

\section{References}

1. Adamo DE, Martin BJ, Brown SH (2007) Age-related differences in upper limb proprioceptive acuity. Percept Mot Skills 104:1297-1309

2. Ahmed AF (2011) Effect of sensorimotor training on balance in elderly patients with knee osteoarthritis. J Adv Res 2:305-311

3. Barrack RL, Skinner HB, Brunet ME, Cook SD (1983) Joint laxity and proprioception in the knee. Phys Sportsmed 11:130-135

4. Chen XY, Chen L, Wolpaw JR (2003) Conditioned H-reflex increase persists after transection of the main corticospinal tract in rats. $\mathrm{J}$ Neurophysiol 90:3572-3578

5. Fischer-Rasmussen T, Jensen PE (2000) Proprioceptive sensitivity and performance in anterior cruciate ligament-deficient knee joints. Scand J Med Sci Sports 10:85-89

6. Fitzpatrick R, McCloskey DI (1994) Proprioceptive, visual and vestibular thresholds for the perception of sway during standing in humans. J Physiol 478:173-186

7. Gardner ER, Martin JH, Jessell TM (2000) The bodily senses. In: Kandel ER, Schwartz JH, Jessell TM (eds) Principles of neural science. McGraw-Hill, New York, pp 430-449

8. Gescheider GA, Thorpe JM, Goodarz J, Bolanowski SJ (1997) The effects of skin temperature on the detection and discrimination of tactile stimulation. Somatosens Mot Res 14:181-188

9. Goble DJ, Coxon JP, Wenderoth N, Van Impe A, Swinnen SP (2009) Proprioceptive sensibility in the elderly: degeneration, functional consequences and plastic-adaptive processes. Neurosci Biobehav Rev 33:271-278

10. Hill KD, LoGiudice D, Lautenschlager NT, Said CM, Dodd KJ, Suttanon P (2009) Effectiveness of balance training exercise in people with mild to moderate severity Alzheimer's disease. BMC Geriatr 9:1-9

11. Hurley MV, Rees J, Newham DJ (1998) Quadriceps function, proprioceptive acuity and functional performance in healthy young, middle-aged and elderly subjects. Age Ageing 27:55-62

12. Iwasaki T, Goto N, Goto J, Ezure H, Moriyama H (2003) The aging of human Meissner's corpuscles as evidenced by parallel sectioning. Okajimas Folia Anat Jpn 79:185-190

13. Ju YY, Liu YC, Cheng HYK, Chang YJ (2011) Rapid repetitive passive movement improves knee proprioception. Clin Biomech 26:188-193

14. Ju YY, Wang CW, Cheng HYK (2010) Effects of active fatiguing movement versus passive repetitive movement on knee proprioception. Clin Biomech 25:708-712

15. Judge JO, King MB, Whipple R, Clive J, Wolfson LI (1995) Dynamic balance in older persons: effects of reduced visual and proprioceptive input. J Gerontol A-Biol 50:M263-M270

16. Kaplan FS, Nixon JE, Reitz M (1985) Age-related changes in proprioception and sensation of joint position. Acta Orthop Scand 56:72-74

17. Kararizou E, Manta P, Kalfakis N, Vassilopoulos D (2005) Morphometric study of the human muscle spindle. Anal Quant Cytol Histol 27:1-4

18. Liu JX, Eriksson PO, Thornell LE, Pedrosa-Domellof F (2005) Fiber content and myosin heavy chain composition of muscle spindles in aged human biceps brachii. J Histochem Cytochem $53: 445-454$
19. Loram ID, Lakie M, Giulio ID, Maganaris CN (2009) The consequences of short-range stiffness and fluctuating muscle activity for proprioception of postural joint rotations: the relevance to human standing. J Neurophysiol 102:460-474

20. Lord SR, Clark RD, Webster IW (1991) Postural stability and associated physiological factors in a population of aged persons. J Gerontol 46:M69-M76

21. Lord SR, Rogers MW, Howland A, Fitzpatrick R (1999) Lateral stability, sensorimotor function and falls in older people. J Am Geriatri Soc 47:1077-1081

22. Lord SR, Sturnieks DL (2005) The physiology of falling: assessment and prevention strategies for older people. J Sci Med Sport $8: 35-42$

23. Lord SR, Ward JA (1994) Age-associated differences in sensorimotor function and balance in community dwelling women. Age Ageing 23:452-460

24. Madhavan S, Shields RK (2005) Influence of age on dynamic position sense: evidence using a sequential movement task. Exp Brain Res 164:18-28

25. McChesney JW, Woollacott MH (2000) The effect of age-related declines in proprioception and total knee replacement on postural control. J Gerontol A-Biol 55:M658-M666

26. McNair PJ, Hewson DJ, Dombroski E, Stanley SN (2002) Stiffness and passive peak force changes at the ankle joint: the effect of different joint angular velocities. Clin Biomech 17:536540

27. National Institutes of Health (2010) Fact sheet - disability in older adults. Retrieved from http://report.nih.gov/NIHfactsheets/Pdfs/ DisabilityinOlderAdults(NIA).pdf. Accessed 10 Nov 2011

28. Nordez A, McNair PJ, Casari P, Cornu C (2009) The effect of angular velocity and cycle on the dissipative properties of the knee during passive cyclic stretching: a matter of viscosity or solid friction. Clin Biomech 24:77-81

29. Pap G, Machner A, Nebelung W, Awiszus F (1999) Detailed analysis of proprioception in normal and ACL-deficient knees. J Bone Joint Surg B 81:764-768

30. Ribeiro F, Oliveira J (2007) Aging effects on joint proprioception: the role of physical activity in proprioception preservation. Eur Rev Aging Phys Act 4:71-76

31. Shaffer SW, Harrison AL (2007) Aging of the somatosensory system: a translational perspective. Phys Ther 87:193-207

32. Skinner HB, Barrack RL, Cook SD (1984) Age related decline in proprioception. Clin Orthop Relat Res 184:208-211

33. Tsang WWN, Hui-Chan CWY (2003) Effects of tai chi on joint proprioception and stability limits in elderly subjects. Med Sci Sports Exerc 35:1962-1971

34. Tsang WWN, Hui-Chan CWY (2004) Effect of 4- and 8-wk intensive tai chi training on balance control in the elderly. Med Sci Sports Exerc 36:648-657

35. Waddington GS, Adams RD (2004) The effect of a 5-week wobble-board exercise intervention on ability to discriminate different degrees of ankle inversion, barefoot and wearing shoes: a study in healthy elderly. J Am Geriatri Soc 52:573-576

36. Westlake KP, Wu Y, Culham EG (2007) Sensory-specific balance training in older adults: effect on position, movement, and velocity sense at the ankle. Phys Ther 87:560-568

37. Wiacek M, Hagner W, Hagner-Derengowska M, Bluj B, Czereba J, Drozd M, Zubrzycki IZ (2009) Deterioration of basic coordinative parameters defines life quality of elderly. Arch Gerontol Geriatr 49:212-214

This research paper is based on actual and original work carried out by the authors. 